\title{
MORBILIDAD DIFERENCIAL ENTRE MUJERES Y HOMBRES
}

\author{
CARMe VAlls-Llobet \\ Especialista en Medicina Interna y Endocrinología
}

Recibido/15/07/2011

Aceptado/25/09/2011

\begin{abstract}
«La investigación de la salud de las mujeres ha sobrepasado los límites que la encerraban solo en los órganos y hormonas relacionados con la reproducción (...)

Este sesgo histórico está siendo redirigido (...)

Cada vez más la asistencia sanitaria se ha de basar en las diferencias biológicas que comporta el sexo y las influencias de género en los factores de conducta, geográficos,culturales, económicos y sociales» ${ }^{1}$
\end{abstract}

\section{Introducción}

El estudio de las diferencias en enfermar entre hombre y mujeres no se ha incorporado hasta la última década del siglo XX. El epígrafe «Women's health» constituye un apartado especifico del Index Medicus a partir del año 1993. Hasta los últimos años del siglo pasado, la mayoría de los conocimientos científicos que se utilizaban para tomar decisiones sobre el diagnóstico y tratamiento de las mujeres se habían basado en estudios que se habían realizado sólo entre hombres. La mayoría de publicaciones y ensayos clínicos hasta el año 1990 no incluían mujeres. Sin embargo los esfuerzos para incluir mujeres en la investigación clínica ha aumentado la información que se ha recopilado en relación a la salud de las mujeres.

1. Pinn VW. Sex and gender factors in medical studies. Implications for health and clinical practice. JAMA. 2003; 289: 397-400.

Feminismo/s 18, diciembre 2011, pp. 281-290 
El Council on Graduate Medical Education y las conclusiones del Primer Congreso sobre Mujeres, Trabajo y Salud realizado en Barcelona en 1996, ya recomendaron que todos los profesionales de la Medicina sea cual sea su especialidad fuera educada a partir de las bases científicas y los conocimientos que se tengan sobre la salud de las mujeres y muestren competencia en proporcionar asistencia médica adecuada a las mujeres. Es esencial que los médicos y médicas conozcan la fisiología básica de las mujeres y la biología reproductiva. Además necesitan apreciar la compleja interacción entre el medio ambiente, la biología y el desarrollo psicosocial de las mujeres. Entre las enfermedades que no sean específicas de las mujeres, los profesionales sanitarios necesitan conocer y ser formados sobre los aspectos de las enfermedades que sean diferentes entre las mujeres o que tengan importantes implicaciones de género.

\section{2 ¿Existen diferencias en el enfermar de mujeres y hombres?}

Tanto la investigación médica, como la docencia y la asistencia sanitaria han mirado a las mujeres «como si fuesen hombres». En los libros de medicina las enfermedades no tienen sexo y como máximo algunas patologías se describen «con mayor frecuencia» entre hombres o entre mujeres. Dado que la ciencia médica ha nacido principalmente en los hospitales, donde la mayoría de patologías son agudas y de predominio masculino, las quejas, el malestar y el dolor de las mujeres, no se ha podido visibilizar, y hasta finales del siglo XX no se empezó a estudiar si existían diferencias en las patologías de mujeres y hombres. La salud de las mujeres se ha estudiado y valorado sólo como salud reproductiva, y este enfoque ha impedido abordar la salud de las mujeres desde una perspectiva integral. También la especialización a que se ha visto sometida la medicina en los últimos treinta años, en aras a la eficacia, ha troceado el cuerpo de los pacientes, y en especial los femeninos, porque son los que presentan más patologías crónicas, que precisan un abordaje multidisciplinar, y su demanda se expresa en los servicios sanitarios ambulatorios ya que en gran medida sus patologías no precisan hospitalización.

La formación del médico o la médica de primaria o de hospital no ha sido enfocada hacia las patologías más prevalentes entre el sexo femenino, ni tampoco dispone, porque no existe, una ciencia sistematizada para apreciar la diferente expresión de la sintomatología de algunas enfermedades según el sexo de las personas. Al faltar formación y docencia específica y mantener en el inconsciente los estereotipos de género, es fácil que los síntomas y patologías más prevalentes entre mujeres hayan permanecido invisibles, sus demandas, síntomas y problemas se hayan considerado de menor importancia, y sus 
repetidas solicitudes de ayuda por estar cansadas o sentir dolor, se han intentado controlar con psicofármacos o con todo tipo de analgésicos sin entender las causas de los síntomas que presenta.

Falta construir un cuerpo de conocimiento ligado a las diferencias por sexo, y que incorpore la perspectiva de género; para lo que se precisa una revisión sistemática de la literatura que rompa estereotipos y proponga las bases a considerar para un diagnóstico diferencial de las enfermedades y del malestar de las mujeres. En definitiva, falta desarrollar el concepto de MORBILIDAD DIFERENCIAL entre mujeres y hombres.

\section{3. ¿Qué significa la morbilidad diferencial entre mujeres y hombres?}

La morbilidad diferencial es el conjunto de enfermedades, motivos de consulta o factores de riesgo que merecen una atención específica hacia las mujeres, sea porque sólo en ellas se pueden presentar dichos problemas o porque sean mucho más frecuentes en el sexo femenino. Puede ser causada por las diferencias biológicas con los hombres, como ocurre con los trastornos de la menstruación, las enfermedades derivadas de embarazos y partos, o los tumores ginecológicos. $\mathrm{O}$, son problemas de salud que sin ser específicos de las mujeres, se presentan con mayor frecuencia (alta prevalencia) en estas, causándoles muerte prematura, enfermedad o discapacidad más que entre los hombres. Es el caso de anemias, dolor crónico, enfermedades autoinmunes, endocrinológicas, ansiedad y depresión.

Existen también factores de riesgo diferenciales que deberían ser prevenidos o abordados de forma diferente en ambos sexos, como las causas de patología cardiovascular, diabetes mellitus, o la obesidad. Lois Verbrugge $e^{2}$ a señalaba en al año 1989 que el peor estado de salud de las mujeres se debía a riesgos adquiridos y aspectos psicosociales, como el sedentarismo, paro, o el estrés físico y mental que produce la doble o triple jornada laboral que deben realizar, así como el trabajo emocional que constantemente realizan en su papel de cuidadoras de toda la familia y de su entorno.

La morbilidad prevalente en el sexo femenino se inicia con los trastornos de la menstruación, que tienen como consecuencia las anemias y las deficiencias en la reserva de hierro. Las enfermedades autoinmunes son de alto predominio femenino y están aumentando en la sociedad industrializada por efectos ambientales y condiciones laborales, y se entrelazan con las enfermedades endocrinológicas, y con carencias metabólicas de enfermedades y vitaminas

2. Verbrugge LM. The twain meet: Empirical explanations of sex differences in health and mortality. J Health Soc Behav. 1989; 30: 282-304. 
por nutrición incorrecta o por diferencias en el metabolismo entre mujeres y hombres, que producen una mayor carencia y más precoz de Vitamina D entre mujeres. Las interacciones biológicas, psicológicas, sociales y medioambientales producen alteraciones en el sistema musculoesquelético dando lugar a las diversas manifestaciones de dolor que dificultan la calidad de vida. Sin dejar de lado las agresiones a la salud mental que supone la violencia de género en toda sus manifestaciones. Este es el cuerpo de la ciencia que falta desarrollar ${ }^{3}$. Después de quince años de trabajo en este tema, intuyo la existencia de intereses creados para que permanezca invisible, ya que existiendo un gran número de información científica sobre las diferentes patologías diferenciales, se minimiza, con el argumento de que son tan frecuentes que es como si fuesen «normales». En consecuencia, se les prescriben psicofármacos por la etiqueta de que son mujeres «ansiosas y depresivas», y no reciben el tratamiento conveniente.

\subsection{De la adolescencia a los 50 años}

Veamos un ejemplo con lo que sucede con los trastornos del ciclo menstrual; así como con las anemias. El equilibrio del ciclo y la ovulación, además de estar influido por la nutrición, lo está por las anemias y las deficiencias de la reserva de hierro, o por alteraciones del resto de glándulas endocrinas. La pérdida de $2 \mathrm{Kg}$ de peso produce en el ciclo menstrual del mes siguiente una disminución de la fase luteínica, y si se sigue perdiendo rápidamente por debajo del Índice de Masa corporal deseable, se pierde primero la ovulación y posteriormente la menstruación. Esta es la causa de amenorrea en la Anorexia Nerviosa.

Las anemias con déficit de glóbulos rojos suponen la enfermedad más prevalente entre el sexo femenino junto con la deficiencia de reservas de hierro. La mayoría de anemias son microcíticas (con glóbulos rojos pequeños) por carencia de hierro, sea por falta de nutrición, por dificultades en su absorción intestinal, o por exceso de sangrados. El 50\% de las mujeres en edad reproductiva y dos terceras partes de mujeres embarazadas en África y en el Sudeste y Oeste asiático están clínicamente anémicas. En Latinoamérica y el Caribe, la anemia entre las mujeres embarazadas es desproporcionadamente alta. Las mujeres, en el tercer trimestre de embarazo y en la lactancia, presentan también altas prevalencia de anemia. El $62,1 \%$ de las mujeres y el $71,4 \%$ de las embarazadas presentaban ferritinas bajas, pero hasta principios del año 2000

3. Valls-Llobet, Carme. 1994. Mujeres y Hombres. Salud y diferencias. Editorial Folio. 4. Valls-Llobet, Carme. 2006. Mujeres Invisibles. Catedra. Colección Feminismos. 
se creía que era «normal» que las mujeres tuvieran ferritinas bajas, y no se habían realizado estudios que correlacionaran los síntomas clínicos con dicha deficiencia. La prevalencia de deficiencia de hierro entre chicas adolescentes oscila entre 9 a $70 \%$ según la población estudiada ${ }^{5}$ frente a solo un 2\% de la población masculina. En edades más avanzadas, entre 70 y 80 años, (75,3 años +- 5) también se mantiene una diferencia significativa de los niveles de ferritina más elevada en hombres que en mujeres como ya demostró el estudio Framingham ${ }^{6}$.

\subsection{Morbilidad por encima de los 50 años.}

La hipertensión diastólica (aumento de la tensión mínima por encima de 10), constituye un importante factor de riesgo de la enfermedad cardiovascular. Durante años la formación de los profesionales sanitarios se había sesgado de forma inconsciente hasta creer que era un factor de riesgo de predominio masculino. Pero diversos estudios han confirmado el predominio femenino entre la población de personas hipertensas. El estudio CARDIA $^{7}$ ha demostrado un predominio de hipertensión entre las personas de raza negra, y de entre ellas en las mujeres. En edades jóvenes el incremento de la hipertensión nocturna y después durante el día, es tres veces más prevalente entre chicas que entre chicos. La prevalencia de enfermedad coronaria (EC) es superior en hombres respecto a mujeres en todos los grupos de $\mathrm{edad}^{8}$. Este hecho ha supuesto la consideración de que la EC es una patología masculina. Pero, la mayor esperanza de vida de las mujeres y, por tanto, su mayor presencia en los grupos de edad más longevos, determina que, en el número absoluto de pacientes con EC, la presencia de mujeres sea mayor. No debemos olvidar que, globalmente hablando, la enfermedad cardiovascular es la primera causa de muerte en mujeres a partir de los 70 años ${ }^{9}$ en España, y la primera causa de muerte en mujeres de EEUU en todas las edades ${ }^{10}$.

5. Bruner AB. Randomised study of cognitive effects of iron supplementation in non-anaemic iron-deficient adolescent girls. Lancet.1996;348:992-996.

6. Fleming DJ, Jacques PF,Dallal GE, Tucker KL, Wilson PWF, Wood RJ. Dietary determinants of iron stores in a free-living elderly population: The Framingham Heart Study. Am J Clin Nutr. 1998; 67:722-733.

7. Levine DA, Lewis CE, Williams OD, Safford MM, Liu K, Calhoun DA, Kim Y, Jacobs D Jr, Kiefe CI.Geographic and demographic variability in 20-year hypertension incidence: theCARDIA study. Hypertension. 2011 Jan;57(1):39-47.

8. Lerner et al. Patterns of coronary heart disease morbidity and mortality in the sexes: a 26-year follow-up of the Framingham population. Am Heart J. 1986; 111(2):383-90.

9. INE. www.ine.es.

10. WHO 1996.

Feminismo/s 18, diciembre 2011, pp. 281-290 
La intervención y el esfuerzo terapéutico sobre la EC en las mujeres es menor que en los hombres en las diferentes etapas de la historia natural de la enfermedad. En prevención primaria, el control de factores de riesgo cardiovasculares (FRCV) es menor, lo que determina que cuando se produce un evento coronario agudo (angina inestable o IAM), las mujeres lleguen en peores condiciones ${ }^{11},{ }^{12}$. Presentan, además, al ser de mayor edad, otras enfermedades al mismo tiempo (comorbilidad) como la Diabetes o la insuficiencia cardíaca $^{13},{ }^{14}$.

La diabetes tipo II o de la adulta/o se presenta también con una alta prevalencia en las mujeres mayores de 40 años, relacionada en muchos casos con la obesidad. La diabetes es una enfermedad de mujeres que se ha estudiado en hombres. El estudio Nurse's Health ${ }^{15}$ ha seguido 84.941 mujeres desde 1980 a 1996, documentando 3300 nuevos casos de diabetes tipo II. El más importante predictor de diabetes fue el sobrepeso o la obesidad (61\%). Otros factores de riesgo demostrados han sido la vida sin ejercicio físico vigoroso, fumar, dieta poco rica en fibra y con exceso de grasa y azúcares y la abstinencia de alcohol (consumo diario inferior a 5 grs) y en últimos trabajos la presencia en sangre de insecticidas, cruzando una vez más en el cuerpo de las mujeres, la contaminación medio ambiental y la salud biológica. Este cruce permanece invisible por los intereses poderosos de la industria química y farmacéutica, y por la debilidad de las políticas públicas que deberían limitar los efectos negativos para la salud de la contaminación industrial, aplicando con rigor, por ejemplo en Europa la iniciativa REACH ${ }^{16}$, para reevaluar la acción sobre la salud de las principales sustancias químicas usadas en la sociedad industrial.

\section{El diagnóstico diferencial del dolor}

El dolor en todas sus manifestaciones corporales es la primera causa de limitación crónica de la actividad principal según la encuesta de salud realizada

11. Hochman et al. Sex, clinical presentation and outcome in patients with acute coronary syndromes. N-England-J-Med. 1999;341:226-232.

12. Vaccarino et al. Sex-based differences in early mortality after myocardial infarction. NEngland-J-Med. 1999;31:217-225.

13. Barrett-Connor et al. Why is diabetes mellitus a stronger risk factor for fatal ischemic heart disease in women than in men?. JAMA. 1991;265:627-631.

14. Austil MA. Epidemiology of hypertrigliceridemia and cardiovascular disease. Am-JCardiol. 1999;83:13F-16F.

15. Hu FB et al. Diet lifestyle and the risk of type 2 diabetes mellitus in women. N Engl J Med.2001; 345:790-797.

16. QUADERN CAPS. SALUT I MEDI AMBIENT. 2008. 
en España ${ }^{17}$, Catalunya ${ }^{18}$ y Barcelona ${ }^{19}$. El dolor constituye la primera causa de consulta en atención primaria y afecta a casi la mitad de los pacientes que buscan asistencia médica ${ }^{20}$. El diagnóstico diferencial del dolor crónico es todavía una asignatura pendiente así como su especial problemática entre el sexo femenino ${ }^{21}$.

Parte de este dolor probablemente tiene su origen en enfermedades autoinmunes, las cuales son más prevalentes entre las mujeres por razones biológicas, hormonales ${ }^{22} \mathrm{y}$, además, por la potenciación del efecto autoinmune que producen los xenoestrógenos (estrógenos exógenos) ambientales ${ }^{23}$.

Se ha podido objetivar también la relación de las condiciones de trabajo y de vida con la presencia de dolor: contractura del músculo trapecio en relación con el estrés físico y mental ${ }^{24}$; dolor lumbar en relación con trabajos con sobre carga emocional ${ }^{25}$ y doble jornada ${ }^{26}$. Está por dilucidar la metodología diagnóstica y la patogénesis del síndrome de fatiga crónica ${ }^{27}$ y de la fibromialgia que se pueden presentar en un 3\% de la población con una razón de mujeres a hombres de $5 / 1$, relacionada también con exposición a tóxicos químicos ambientales ${ }^{28}$.

\section{Conclusión}

El sesgo de género en atención sanitaria se ha debido a la falta de investigación en morbilidad diferencial y a la visión androcéntrica que tiene la medicina

17. Encuesta Nacional de Salud.Ministerio de Sanidad y Consumo 1988.

18. Enquesta de Salut de Catalnya. Departament de Sanitat i Seguretat Social, Generalitat de Catalunya, 1995.

19. Enquesta de Salut de Barcelona. Ajuntament de Barcelona 1996.

20. Griggs RC et al. Muscle pain, fatigue, and mitochondriopathies. $N$ Engl J Med. 1999;341(14):1077-8.

21. Valls Llobet C. Diagnóstico diferencial del dolor y fatiga crónica: una asignatura pendiente. Med Clin. 2003;118(1).

22. Greenstein BD. Lupus: why women? J Womens Health Gend Based Med. 2001 Apr; 10(3):233-9.

23. Olea N. Disruptores endocrinos: posibles medidas de inervención. La perspectiva europea. Quadern Caps. 2000;29:36-42.

24. Lundberg U. Influence of paid and unpaid work on psychophysiological stress. Responses of men and women. J-Ocupational-Health.1996;1(2):117-130.

25. Deyo RA et al. Low back. N Engl J Med. 2001;344(5):363-370.

26. Simon GE. An international study of the relation between somatic simptoms and depression. N Engl J Med. 1999;341(18):1329-1335.

27. Shafran SD. The chronic fatigue syndrome. Am J Med.1991;90:730-738.

28. Bell IR et al. Illness from low levels of environmental chemicals: relevance to chronic fatigue syndrome and fibromyalgia. Am J Med.1998;105(3A):74S-82-S.

Feminismo/s 18, diciembre 2011, pp. 281-290 
como norma ${ }^{29}$. La rigidez de la visión centrada sólo en el hombre ha producido sesgos en el momento del diagnóstico, tanto por considerar que enferman igual hombres y mujeres, cuando hay diferencias, como por considerar, inversamente, que como son diferentes no pueden tener problemas similares ${ }^{30}, \mathrm{o}$ bien por no considerar los condicionantes bio-psico-sociales de las enfermedades $^{31}{ }^{1}$. Además de los sesgos en los diagnósticos, también se han producido sesgos de género en los métodos de exploración, en la valoración de la normalidad en los análisis clínicos, y en la aplicación de terapias sin ninguna diferenciación por sexo, ni en dosis ni en vía administrada. El feminismo como defensa de los derechos de las mujeres no puede permanecer indiferente hacia la desigualdad de oportunidades que supone no tener en cuenta en el momento del diagnóstico médico, las diferencias en el enfermar de las mujeres respecto a los hombres, y debería potenciar en Medicina la producción de conocimiento de la morbilidad diferencial entre ambos sexos.

\section{Referencias Bibliográficas}

AUSTIL MA. Epidemiology of hypertrigliceridemia and cardiovascular disease. Am-J-Cardiol. (1999);83:13F-16F

BARRETT-CONNOR et al. ¿Why is diabetes mellitus a stronger risk factor for fatal ischemic heart disease in women than in men?. JAMA. (1991);265:627-631

BELL IR et al. Illness from low levels of environmental chemicals: relevance to chronic fatigue syndrome and fibromyalgia. Am J Med. (1998);105(3A):74S-82-S

BRUNER AB. «Randomised study of cognitive effects of iron supplementation in non-anaemic iron-deficient adolescent girls». Lancet. (1996); 348:992-996

CI.Geographic and demographic variability in 20-year hypertension incidence: the CARDIA study. Hypertension. (2011) Jan; 57(1):39-47

DeYo RA Weinstein, J.N. Low back. N Engl J Med. (2001);344(5):363-370

Fleming DJ, JacQues PF, Dallal GE, TuCKer KL, Wilson PWF, WoOd RJ. Dietary determinants of iron stores in a free-living elderly population: The Framingham Heart Study. Am J Clin Nutr. (1998); 67:722-733

29. Mastroianni AC, Faden R, Federman D. eds.Women and Health Research: Ethical and Legal Issues of Including Women in Clinical Studies. Washington, DC: National Academy Press; 1994.

30. Ruiz MT, Verbrugge LM A two way view of gender bias in medicine. J-Epidemiol-Community-Health.(1997);51:106-109.

31. Valls-Llobet, C. El estado de la investigación en salud y género. En «Perspectivas de género en salud. Fundamentos cientificos y socioprofesionales de diferencias sexuales no previstas. Grupo de Salud del Saminario Interdisciplinar de Estudios de la Mujer de la Universidad de Zaragoza. Minerva Ediciones. 2001. 
GREENSTEIN BD. Lupus: ¿why women? J Womens Health Gend Based Med. (2001) Apr; 10 (3):233-9.

GRIGGS RC et al. Muscle pain, fatigue, and mitochondriopathies. N Engl J Med. (1999); 341(14):1077-8

VAlls Llobet C. Diagnóstico diferencial del dolor y fatiga crónica: una asignatura pendiente. Med Clin. (2003) 1;118

Hochman Judith S. M.D., Jacqueline E. TAmis, M.D., Trevor D. Thompson, B.S., W. Douglas Weaver, M.D., Harvey D. White, M.B., D.Sc., Frans Van DE Werf, M.D., Phil Aylward, B.M., B.Ch., Eric J. Topol, M.D., and Robert M. CALIFF, M.D. Sex, clinical presentation and outcome in patients with acute coronary syndromes. N-England-J-Med. (1999);341:226-232.

Hu FB, Manson, J.E., Stampfer, M.J. Diet lifestyle and the risk of type 2 diabetes mellitus in women. N Engl J Med(.2001); 345:790-797

Levine DA, Lewis CE, Williams OD, SafFord MM, Liu K, Calhoun DA, Kim Y, JACOBS D Jr, KIEFE

LERNER et al. Patterns of coronary heart disease morbidity and mortality in the sexes: a 26-year follow-up of the Framingham population. Am Heart J. 1986; 111(2):383-90.

LUNDBERG U. Influence of paid and unpaid work on psychophysiological stress. Responses of men and women. J-Ocupational-Health. (1996); 1(2):117-130

Mastroianni AC, Faden R, Federman D. (Eds).»Women and Health Research: Ethical and Legal Issues of Including Women in Clinical Studies». Washington, DC: National Academy Press; 1994.

OlEA N. Disruptores endocrinos: posibles medidas de inervención. La perspectiva europea. Quadern Caps. 2000;29:36-42

PINN VW. Sex and gender factors in medical studies. Implications for health and clinical practice. JAMA. (2003); 289: 397-400.

RUIZ MT, VERBRUGGE LM A two way view of gender bias in medicine. J-EpidemiolCommunity-Health.(1997);51:106-109

SHAFRAN SD. The chronic fatigue syndrome. Am J Med.1991;90:730-738

SIMON GE. An international study of the relation between somatic simptoms and depression. N Engl J Med. (1999);341(18):1329-1335

VACCARINO, V. Sex-based differences in early mortality after myocardial infarction. N-England-J-Med. (1999);31:217-225.

Valls-Llobet, Carme. Mujeres Y Hombres. Salud y diferencias.Editorial Folio, 1994.

VAlls-Llobet, Carme. El estado de la investigación en salud y género. En «Perspectivas de género en salud. Fundamentos cientificos y socioprofesionales de diferencias sexuales no previstas». Grupo de Salud del Saminario Interdisciplinar de Estudios de la Mujer de la Universidad de Zaragoza. Minerva Ediciones. 2001 
VAlls-Llobet, Carme.Mujeres Invisibles. Catedra. Colección Feminismos. 2006

VerbrugGe LM. The twain meet: Empirical explanations of sex differences in health and mortality. J Health Soc Behav. (1989); 30: 282-304. 